OPEN ACCESS

Edited by:

Alberto Di Leo,

Ospedale San Camillo, Italy

Reviewed by:

Jun Lyu,

First Affiliated Hospital of Jinan

University, China

Ning Pu,

Fudan University, China

Marco Milone

Federico II University Hospital, Italy

*Correspondence:

Chundong Zhang

zhangchundong2007@126.com;

czhang@ncc.go.jp

Rui Zhang

zhangrui@cancerhosp-In-cmu.com

Ji Zhu

leoon.zhu@gmail.com

${ }^{t}$ These authors have contributed equally to this work and share first authorship

Specialty section:

This article was submitted to

Surgical Oncology,

a section of the journal

Frontiers in Oncology

Received: 21 August 2020

Accepted: 12 October 2020

Published: 03 November 2020

Citation:

Zhang N, Ning F, Guo R, Pei J, Qiao Y,

Fan J, Jiang B, Liu Y, Chi Z, Mei Z, Abe $M$, Zhu J, Zhang $R$ and Zhang $C$

(2020) Prognostic

Values of Preoperative Inflammatory and Nutritional Markers for

Colorectal Cancer.

Front. Oncol. 10:585083.

doi: 10.3389/fonc.2020.585083

\section{Prognostic Values of Preoperative Inflammatory and Nutritional Markers for Colorectal Cancer}

\author{
Nannan Zhang ${ }^{1 \dagger}$, Feilong Ning ${ }^{2 \dagger}$, Rui Guo ${ }^{3 \dagger}$, Junpeng Pei ${ }^{4}$, Yun Qiao ${ }^{3}$, Jin Fan $^{5,6}$, \\ Bo Jiang ${ }^{7}$, Yanlong Liu ${ }^{8}$, Zhaocheng Chi ${ }^{9}$, Zubing Mei ${ }^{10,11}$, Masanobu Abe ${ }^{12}$, Ji Zhu ${ }^{5,6^{*}}$, \\ Rui Zhang ${ }^{3 *}$ and Chundong Zhang ${ }^{4,13^{*}}$
}

\begin{abstract}
1 State key Laboratory of Cancer Biology and National Clinical Research Center for Digestive Diseases, Xijing Hospital of Digestive Diseases, Fourth Military Medical University, Xi'an, China, ${ }^{2}$ Department of General Surgery, Xuzhou Hospital of Traditional Chinese Medicine, Xuzhou, China, ${ }^{3}$ Department of Colorectal Surgery, Cancer Hospital of China Medical University, Liaoning Cancer Hospital \& Institute, Shenyang, China, ${ }^{4}$ Department of Gastrointestinal Surgery, The Fourth Affiliated Hospital of China Medical University, Shenyang, China, ${ }^{5}$ Department of Radiation Oncology, Fudan University Shanghai Cancer Center, Shanghai, China, ${ }^{6}$ Department of Oncology, Shanghai Medical College of Fudan University, Shanghai, China, ${ }^{7}$ Department of Colorectal Anal Surgery, Shanxi Province Cancer Hospital \& Institute, Taiyuan, China, ${ }^{8}$ Department of Colorectal Surgery, Harbin Medical University Cancer Hospital, Harbin, China, ${ }^{9}$ Second Department of Gastrointestinal Surgery, Jilin Cancer Hospital, Changchun, China, ${ }^{10}$ Department of Anorectal Surgery, Shuguang Hospital, Shanghai University of Traditional Chinese Medicine, Shanghai, China, ${ }^{11}$ Department of Anorectal Surgery, Anorectal Disease Institute of Shuguang Hospital, Shanghai, China, ${ }^{12}$ Division for Health Service Promotion, University of Tokyo, Tokyo, Japan, ${ }^{13}$ Department of Gastrointestinal Surgery, Graduate School of Medicine, University of Tokyo, Tokyo, Japan
\end{abstract}

Background: Increasing evidence indicates that inflammation and nutritional status are associated with survival outcomes in patients with colorectal cancer (CRC). This study aimed to investigate the prognostic values of preoperative inflammatory and nutritional factors and develop a prognostic model individually predicting overall survival (OS) and disease-free survival (DFS) in patients with CRC.

Methods: We retrospectively collected data on patients with CRC who underwent radical surgery. Independent prognostic inflammatory and nutritional markers were identified and novel prognostic models were developed incorporating the identified factors. The discriminative ability and model-fitting performance were evaluated by receiver operating characteristic curves and Akaike information criteria. Clinical usefulness was assessed by decision curve analysis.

Results: A total of 400 eligible patients were identified. Multivariate analysis identified pN stage, tumor differentiation grade, neutrophil count, and body mass index as independent prognostic factors for OS, and $\mathrm{pN}$ stage, tumor differentiation grade, neutrophil count, neutrophil-lymphocyte ratio, and serum albumin as prognostic factors for DFS. The combined inflammatory and nutritional prognostic model showed better discriminative ability, model-fitting performance, and net benefits than the inflammatory and nutritional models alone, and the American Joint Committee on Cancer (AJCC) 8th TNM classification for predicting OS and DFS. 


\begin{abstract}
Conclusion: Preoperative nutritional and inflammatory factors have significant prognostic value in patients with $\mathrm{CRC}$. A novel prognostic model incorporating preoperative inflammatory and nutritional markers provides better prognostic performance than the AJCC 8th TNM classification. A novel nomogram incorporating preoperative inflammatory and nutritional markers can individually predict OS and DFS in patients with CRC.
\end{abstract}

Keywords: colorectal cancer, inflammatory status, nutritional status, prognostic model, survival outcome

\section{INTRODUCTION}

Colorectal cancer (CRC) is a significant global health burden. It is the third most common cancer in Western countries with more than 140,000 new cases diagnosed in the United States in 2019 and is one of the leading causes of cancer related deaths, with around 700,000 deaths reported globally each year $(1,2)$. Survival outcomes of advanced CRC have improved as a result of recent advances in surgery, systemic therapy, and best supportive care. The gold standard for predicting survival and surveillance recommendations for CRC remains the Union for International Cancer Control/American Joint Committee on Cancer (UICC/ AJCC) tumor/node/metastasis (TNM) anatomical classification (3). However, other factors, including tumor size (4), tumor location (5), tumor deposits (6), lymph node ratio (7), lymphovascular infiltration (8), and carcinoembryonic antigen level (9), have also been associated with patient prognosis and may complement anatomical TNM staging.

Preoperative systemic inflammatory factors play crucial roles in the carcinogenesis and progression of CRC (10) and were proposed as predictors of recurrence and prognostic factors for overall survival (OS) and disease-free survival (DFS) (11). Inflammatory factors, including neutrophil count (12), lymphocyte count (13), neutrophil-lymphocyte ratio (NLR) (14), lymphocyte-monocyte ratio (LMR) (15), and plateletlymphocyte ratio (PLR) (16) were shown to be independently associated with prognosis in patients with CRC.

Preoperative nutritional factors were also associated with postoperative morbidity and mortality $(17,18)$, and can be applied as prognostic markers of CRC. Some preoperative nutritional markers, including body mass index (BMI) (19), serum albumin (20), loss of muscle mass (21), and prognostic nutritional index (22) have demonstrated prognostic value in patients with CRC. Thus, some preoperative inflammatory and nutritional factors could predict survival outcomes and might be used to develop a novel prognostic model for patients with CRC.

In this study, we aimed to investigate the prognostic values of preoperative inflammatory and nutritional markers in patients with curable CRC. In addition, we aimed to develop a novel prognostic model incorporating relevant preoperative inflammatory and nutritional markers for the individual prediction of survival outcomes in patients with CRC. We compared the predictive performances of this novel prognostic model with the AJCC 8th TNM classification in terms of model discriminative ability, modelfitting performance, and clinical utility.

\section{PATIENTS AND METHODS}

\section{Data Source}

We retrospectively collected data on patients with CRC who underwent radical surgery at the Department of Colorectal Surgery, Cancer Hospital of China Medical University, Liaoning Cancer Hospital and Institute. The study was approved by the Ethics Committee of the Cancer Hospital of China Medical University, Liaoning Cancer Hospital and Institute, and written informed consent was obtained from all patients.

\section{Eligibility Criteria}

Patients were included according to the following criteria: (1) pathologically confirmed primary CRC; (2) no other synchronous malignancy; (3) sufficient data regarding the analyzed clinicopathological characteristics; (4) no distant metastasis (M0) before operation; (5) no preoperative treatment (radiotherapy and/or chemotherapy); (6) no history of cancer surgery; (7) pathologically negative resection margins (R0 resection); and (8) postoperative survival $\geq 1$ month. Preoperative neoadjuvant therapy may have complex and profound impacts on inflammatory and nutritional factors, and we therefore only explored the prognostic values of preoperative inflammatory and nutritional markers in patients with curable CRC who had not received preoperative neoadjuvant therapy.

\section{Patient Management}

Radical en bloc resection was applied in all patients with curable CRC. All pathological specimens were analyzed independently by two experienced pathologists and disagreements were resolved through discussions. Follow-ups were conducted every 3-6 months for the first 2 years, every 6 months for the next 3 years, and annually thereafter until January 2020 or the time of death. Two doctors were responsible for all follow-ups and for recording all the information. The date of last follow-up was January 2020. OS was defined as the time from the date of surgery to death from any cause, and DFS was defined as the time from surgery to the identification of disease recurrence (23).

\section{Main Outcomes}

The clinical and pathological indexes included history of smoking and drinking, sex, age, operation type, tumor location, tumor size, tumor differentiation grade, number of retrieved lymph nodes, AJCC 8th pathological T stage (pT stage), AJCC 8th pathological $\mathrm{N}$ stage ( $\mathrm{pN}$ stage), vascular invasion, perineural invasion, and adjuvant therapy. Inflammatory and laboratory indexes included 
white blood cell (WBC) count, neutrophil count, lymphocyte count, platelets, NLR, LWR, PLR, neutrophil-WBC ratio (NWR), and platelet-neutrophil ratio (PNR). The nutritional indexes included serum albumin, hemoglobin, and BMI.

\section{Statistical Analysis}

Categorical data were shown as number (percentage) and continuous data were presented as mean ( \pm standard deviation). Differences in OS and DFS were assessed by Kaplan-Meier survival analysis with log-rank tests. Factors with a $P$ value $<0.1$ in univariate analyses were considered as potential prognostic factors $(24,25)$ and were incorporated into Cox proportional hazards regression models, and hazard ratios (HRs) with 95\% confidence intervals (CIs) were presented.

The identified independent prognostic factors were applied as basic indexes in the novel prognostic models. We then proposed the following novel prognostic models based on the independent prognostic factors identified by Cox proportional hazards regression models as follows: (1) basic indexes incorporating preoperative inflammatory markers; (2) basic indexes incorporating preoperative nutritional markers; and (3) basic indexes incorporating both preoperative inflammatory and nutritional markers. The AJCC 8th TNM classification was applied as the control model.

The predictive performances of the novel prognostic models were compared in terms of model discriminative ability, modelfitting performance, and clinical utility. Area under the receiver operating characteristic curve (AUC, ROC) analysis was performed to assess model discriminative ability, and Akaike information criteria (AIC) and concordance index (C-index) analyses were carried out to assess model-fitting performance (26). A higher AUC value demonstrated superior model discriminative ability, and a higher C-index or lower AIC value revealed better modelfitting performance. Calibration plots were applied to assess agreements between the predicted and actual probabilities of 3- or 5 -year OS and DFS. Decision curve analyses (DCAs) were further applied to measure and compare the clinical utilities of the different prognostic models (27). Novel prognostic nomograms were developed for the individual prediction of survival outcomes.

All statistical analyses were performed using SPSS version 22.0 and $\mathrm{R}$ version 3.6.3. All tests were two-sided and a $\mathrm{P}$ value $<$ 0.05 was considered statistically significant.

\section{RESULTS}

\section{Basic Characteristics}

A total of 400 patients with CRC were finally included in the study. The clinicopathological characteristics of the included patients are shown in Table 1. There were 171 patients with colon cancer and 229 with rectal cancer. The distribution of AJCC 8th TNM classification was 59 patients (14.8\%) in stage I, $181(45.3 \%)$ in stage II, and $160(40.0 \%)$ in stage III. The pT stage distribution of the 229 patients with rectal cancer was as follows: 11 pT1 cases, 39 pT2 cases, 117 pT3, and 62 pT4 cases. The median follow-up time was 31 months (range, 1-74 months).

\section{Independent Prognostic Factors}

Univariate analysis identified history of drinking, tumor location, tumor size, tumor differentiation grade, $\mathrm{pT}$ stage, $\mathrm{pN}$ stage, and adjuvant therapy as potential prognostic factors for OS (log-rank tests, all $\mathrm{P}<0.10)$ (Table 2). Tumor differentiation grade ( $\mathrm{HR}$ 2.12; 95\% CI, 1.28-3.53; P = 0.004) and $\mathrm{pN}$ stage (HR 1.61; 95\% CI, 1.39-1.85; $\mathrm{P}<0.001$ ) were further identified as independent prognostic factors for OS by multivariate analysis (Table 2).

Univariate analysis identified a history of drinking, operation type, tumor size, tumor differentiation grade, $\mathrm{pT}$ stage, $\mathrm{pN}$ stage, and adjuvant therapy as potential prognostic factors for DFS (logrank tests, all $\mathrm{P}<0.10$ ) (Table 3), and tumor differentiation grade (HR 2.25; 95\% CI, 1.43-3.52; P < 0.001), pT stage (HR 1.52; 95\% CI, 1.12-2.06; $\mathrm{P}=0.008$ ), and $\mathrm{pN}$ stage (HR 1.53; 95\% CI, 1.35-1.74; $\mathrm{P}<0.001$ ) were further identified as independent prognostic factors for DFS by multivariate analysis (Table 3). These identified prognostic factors were applied as basic indexes in the novel prognostic models.

\section{Preoperative Inflammatory and Nutritional Markers}

The preoperative inflammatory markers of WBC count, neutrophil count, NWR, LWR, and PNR were identified as potential predictive factors for OS, and WBC count, neutrophil count, NWR, NLR, LWR, PNR, and PLR as potential predictive factors for DFS by univariate analyses (log-rank tests, all $\mathrm{P}<0.10$ ) (Table 4). The preoperative nutritional markers hemoglobin, serum albumin, and BMI were potential predictive factors for both OS and DFS (log-rank tests, all $\mathrm{P}<0.10$ ) (Table 4).

\section{Development of Novel Prognostic Models}

We developed an inflammatory model predicting OS including tumor differentiation grade, $\mathrm{pN}$, stage and neutrophil count, and an inflammatory model predicting DFS including $\mathrm{pT}$ stage, $\mathrm{pN}$ stage, tumor differentiation grade, neutrophil count, and NLR (Tables 5 and 6). We also developed a nutritional model predicting OS including $\mathrm{pN}$ stage, tumor differentiation grade, and BMI, and a nutritional model predicting DFS including $\mathrm{pN}$ stage, tumor differentiation grade, and serum albumin (Tables 5 and 6). The combined inflammatory and nutritional model predicting OS included $\mathrm{pN}$ stage, tumor differentiation grade, neutrophil count, and BMI, and the equivalent model predicting DFS included $\mathrm{pN}$ stage, tumor differentiation grade, neutrophil count, NLR, and serum albumin (Tables 5 and 6 ).

\section{Assessment of Predictive Performance}

The combined inflammatory and nutritional model showed superior model discriminative ability in terms of OS (AUC, 0.820 ; 95\% CI, 0.749-0.890) compared with the inflammatory model (AUC, 0.796; 95\% CI, 0.72-0.872), nutritional model (AUC, 0.793; 95\% CI, 0.722-0.864), and the AJCC 8th TNM classification (AUC, 0.789; 95\% CI, 0.716-0.862) (Figure 1 and Table 2). It also showed better model-fitting performance (C-index, 0.813; AIC, 513.6) than the inflammatory model (C-index, 0.794; AIC, 522.6), nutritional model (C-index, 
TABLE 1 | Clinical, pathological, laboratory and nutritional characteristics.

\begin{tabular}{|c|c|c|c|}
\hline Variables & Value & Variables & Value \\
\hline Clinical factors & & No. of positive LNs & $1.80( \pm 4.61)$ \\
\hline Age (years) & $60.7( \pm 11.2)$ & AJCC $8^{\text {th }} \mathrm{pN}$ stage & \\
\hline Gender & & pNO & $240(60.0 \%)$ \\
\hline Male & 238 (59.5\%) & $\mathrm{pN} 1 \mathrm{a}$ & 60 (15.0\%) \\
\hline Female & $162(40.5 \%)$ & $\mathrm{pN} 1 \mathrm{~b}$ & $41(10.3 \%)$ \\
\hline History of smoking & & $\mathrm{pN} 1 \mathrm{c}$ & $5(1.3 \%)$ \\
\hline Yes & $93(23.3 \%)$ & $\mathrm{pN} 2 \mathrm{a}$ & $24(6.0 \%)$ \\
\hline No & 307 (76.8\%) & $\mathrm{pN} 2 \mathrm{~b}$ & $30(7.5 \%)$ \\
\hline History of drinking & & AJCC $8^{\text {th }}$ pTNM stage & \\
\hline Yes & $96(24.0 \%)$ & 1 & $59(14.8 \%)$ \\
\hline No & 304 (76.0\%) & $\| A$ & 127 (31.8\%) \\
\hline Operation type & & $\| \mathrm{B}$ & 45 (11.3\%) \\
\hline Laparoscopic surgery & $56(14.0 \%)$ & $\| \mathrm{C}$ & $9(2.3 \%)$ \\
\hline Open surgery & 344 (86.0\%) & $\| \mathrm{A}$ & $6(1.5 \%)$ \\
\hline Adjuvant therapy & & IIIB & $104(26.0 \%)$ \\
\hline Yes & 219 (54.8\%) & $I I I C$ & $50(12.5 \%)$ \\
\hline No & $181(45.3 \%)$ & Vascular invasion & \\
\hline Pathological factors & & Negative & 340 (85.0\%) \\
\hline Tumor location & & Positive & $60(15.0 \%)$ \\
\hline Colon & $171(42.8 \%)$ & Perineural invasion & \\
\hline Rectum & 229 (57.3\%) & Negative & 380 (95.0\%) \\
\hline Tumor size (cm) & $4.69( \pm 1.86)$ & Positive & $20(5.0 \%)$ \\
\hline Tumor differentiation grade & & Inflammatory/laboratory factors & \\
\hline Well differentiation & $33(8.3 \%)$ & WBC $\left(\times 10^{9} / \mathrm{L}\right)$ & $6.61( \pm 2.07)$ \\
\hline Moderate differentiation & $326(81.5 \%)$ & Neut $\left(\times 10^{9} / \mathrm{L}\right)$ & $4.13( \pm 2.12)$ \\
\hline Poorly differentiation & $37(9.3 \%)$ & $\operatorname{Lym}\left(\times 10^{9} / \mathrm{L}\right)$ & $1.87( \pm 1.71)$ \\
\hline Undifferentiation & $4(1.0 \%)$ & Plt $\left(\times 10^{9} / \mathrm{L}\right)$ & $268.5( \pm 89.0)$ \\
\hline No. of retrieved LNs (\%) & $16.2( \pm 9.64)$ & NWR & $58.7( \pm 20.1)$ \\
\hline Adequate $(n \geq 12)$ & $253(63.2 \%)$ & NLR & $2.81( \pm 4.30)$ \\
\hline Inadequate $(\mathrm{n}<12)$ & $147(36.8 \%)$ & LWR & $27.2( \pm 9.76)$ \\
\hline AJCC $8^{\text {th }} \mathrm{pT}$ stage & & PNR & $109.8( \pm 175.5)$ \\
\hline pT1 & 17 (4.3\%) & PLR & $171.4( \pm 126.7)$ \\
\hline рT2 & $51(12.8)$ & Nutritional factors & \\
\hline рT3 & 212 (53.0\%) & $\mathrm{Hgb}(\mathrm{g} / \mathrm{L})$ & $135.3( \pm 23.2)$ \\
\hline pT4a & 95 (23.8\%) & Alb (g/L) & $42.2( \pm 5.43)$ \\
\hline $\mathrm{pT} 4 \mathrm{~b}$ & $25(6.3 \%)$ & $\mathrm{BMl}\left(\mathrm{kg} / \mathrm{m}^{2}\right)$ & $23.5( \pm 3.31)$ \\
\hline
\end{tabular}

AIC, Akaike's Information Criterion; Alb, albumin; AJCC, American Joint Committee on Cancer; BMI, body mass index; Hgb, hemoglobin; LWR, Iymphocyte to white blood cell ratio; Lym, lymphocyte count; LNs, lymph nodes; Neut, neutrophil count; No., number; NLR, neutrophil to lymphocyte ratio; NWR, neutrophil to white blood cell ratio; PLR, platelet to lymphocyte ratio; Plt, platelet count; PNR, platelet to neutrophil ratio; WBC, white blood cell count.

0.782; AIC, 521.5), and AJCC 8th TNM classification (C-index, 0.776; AIC, 535.3) (Table 5).

Similar comparative results were found in terms of modelfitting performance and model discriminative ability for DFS (Table 6 and Figure 1). Furthermore, the calibration plots of all models showed good agreement between the predicted and actual probabilities of 3- or 5-year OS and DFS (Figure 2).

\section{Clinical Utility Estimated by DCA}

DCA was performed to estimate the clinical utilities of the novel prognostic models and the control AJCC 8th TNM classification. The combined inflammatory and nutritional model revealed superior net benefits over the inflammatory and nutritional models alone in terms of predicting 3- and 5-year OS between the threshold probabilities of $15 \%-45 \%$ and $20 \%-55 \%$, respectively (Figures 3A, B). All three novel prognostic models showed superior net benefits compared with the AJCC 8th TNM classification for predicting 3- and 5-year OS between the threshold probabilities of $15 \%-60 \%$ and $20 \%-60 \%$, respectively. Similar findings were obtained for DCAs predicting 3- and 5-year DFS (Figures 3C, D).

\section{Nomograms Individually Predicting Survival}

A combined inflammatory and nutritional nomogram including $\mathrm{pN}$ stage, tumor differentiation grade, neutrophil count, and BMI was established for individually predicting 1-, 3-, and 5-year OS (Figure 4A), and a combined nomogram including $\mathrm{pN}$ stage, tumor differentiation grade, neutrophil count, NLR, and serum albumin was established for individually predicting 1-, 3-, and 5year DFS (Figure 4B). Inflammatory and nutritional nomograms were also established (Figures $\mathbf{5}$ and $\mathbf{6}$ ).

\section{DISCUSSION}

Increasing evidence has demonstrated decisive roles for inflammatory and nutritional indexes in the prognosis of 
TABLE 2 | Univariate and multivariate analyses of prognostic factors for overall survival.

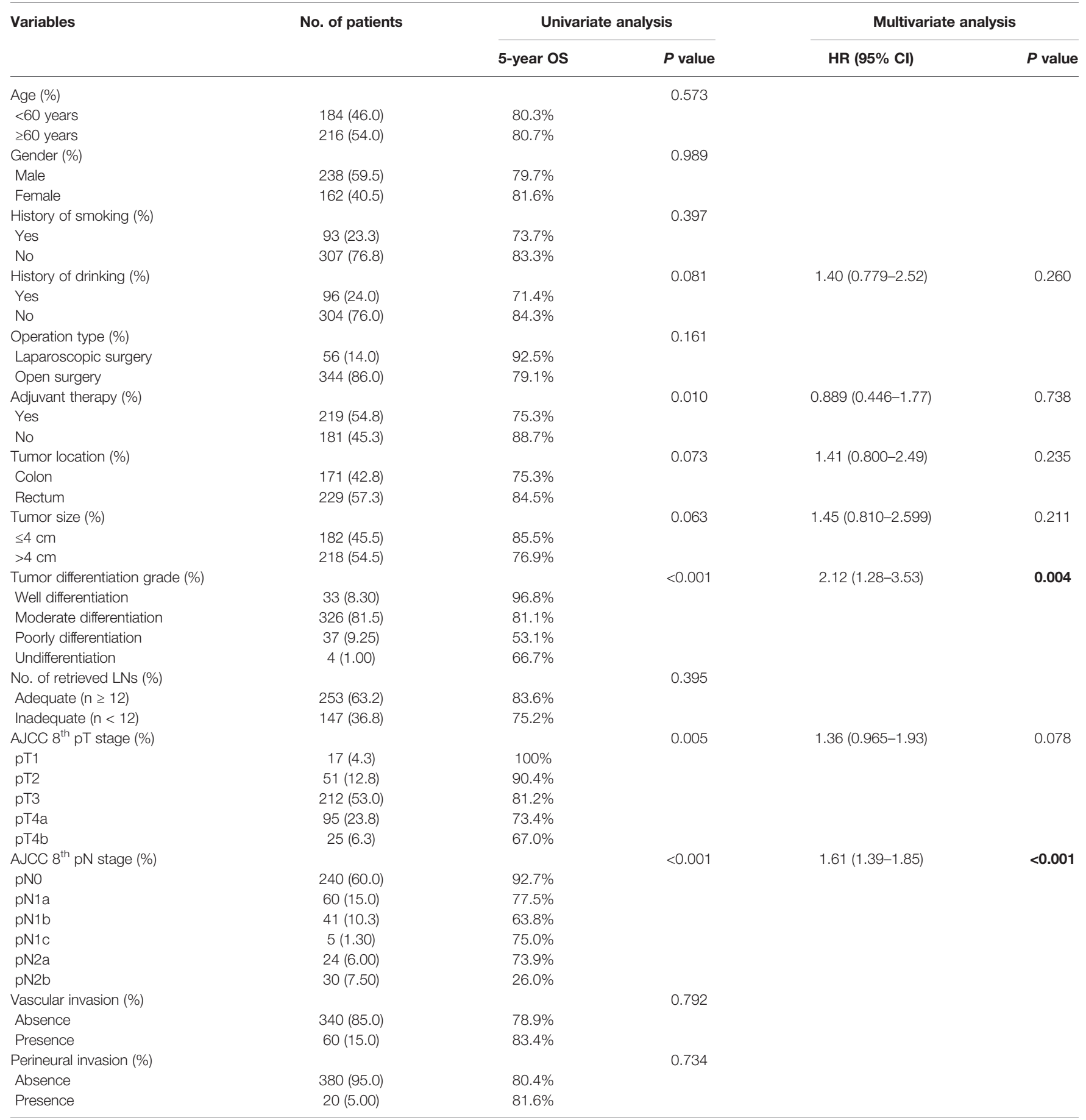

AJCC, American Joint Committee on Cancer; Cl, confidence interval; HR, hazard ratio; LNs, lymph nodes; No., number. Significant values $(P<0.05)$ are in bold.

cancers, including CRC $(10,17,18)$. Although the AJCC TNM anatomical classifications have been regarded as the most robust prognostic indicator for CRC (3), systemic inflammation and nutritional markers can provide additional prognostic value to complement this classification (3). We therefore investigated the associations between preoperative systemic inflammation and nutritional statuses and survival outcomes in patients with CRC. We further developed novel prognostic models based on identified independent nutritional and inflammatory prognostic factors for individually predicting OS and DFS in patients with CRC undergoing curative surgery.

Systemic inflammation caused by complicated host-tumor interactions is an important component of tumors and plays a pivotal role in cancer initiation, development, and metastasis (28, 29). Many inflammatory parameters have been reported to be independently associated with the prognosis of CRC, including 
TABLE 3 | Univariate and multivariate analyses of prognostic factors for disease-free survival.

\begin{tabular}{|c|c|c|c|c|c|}
\hline \multirow[t]{2}{*}{ Variables } & \multirow[t]{2}{*}{ No. of patients } & \multicolumn{2}{|c|}{ Univariate analysis } & \multicolumn{2}{|c|}{ Multivariate analysis } \\
\hline & & 5-year DFS & $P$ value & HR $(95 \% \mathrm{Cl})$ & $P$ value \\
\hline Age (\%) & & & 0.665 & & \\
\hline$<60$ years & $184(46.0)$ & $77.8 \%$ & & & \\
\hline$\geq 60$ years & $216(54.0)$ & $78.1 \%$ & & & \\
\hline Gender (\%) & & & 0.598 & & \\
\hline Male & $238(59.5)$ & $76.0 \%$ & & & \\
\hline Female & $162(40.5)$ & $80.9 \%$ & & & \\
\hline History of smoking (\%) & & & 0.375 & & \\
\hline Yes & $93(23.3)$ & $70.1 \%$ & & & \\
\hline No & $307(76.8)$ & $81.1 \%$ & & & \\
\hline History of drinking (\%) & & & 0.097 & 1.32 (0.776-2.24) & 0.307 \\
\hline Yes & $96(24.0)$ & $67.9 \%$ & & & \\
\hline No & $304(76.0)$ & $82.1 \%$ & & & \\
\hline Operation type (\%) & & & 0.049 & $0.661(0.194-2.26)$ & 0.508 \\
\hline Laparoscopic surgery & $56(14.0)$ & $92.5 \%$ & & & \\
\hline Open surgery & $344(86.0)$ & $76.1 \%$ & & & \\
\hline Adjuvant therapy (\%) & & & 0.002 & $0.843(0.443-1.60)$ & 0.603 \\
\hline Yes & $219(54.8)$ & $71.9 \%$ & & & \\
\hline No & $181(45.3)$ & $86.6 \%$ & & & \\
\hline Tumor location (\%) & & & 0.116 & & \\
\hline Colon & $171(42.8)$ & $75.5 \%$ & & & \\
\hline Rectum & $229(57.3)$ & $82.3 \%$ & & & \\
\hline Tumor size (\%) & & & 0.069 & $1.31(0.779-2.21)$ & 0.309 \\
\hline$\leq 4 \mathrm{~cm}$ & $182(45.5)$ & $82.9 \%$ & & & \\
\hline$>4 \mathrm{~cm}$ & $218(54.5)$ & $74.4 \%$ & & & \\
\hline Tumor differentiation grade (\%) & & & $<0.001$ & $2.25(1.43-3.52)$ & $<0.001$ \\
\hline Well differentiation & $33(8.30)$ & $96.8 \%$ & & & \\
\hline Moderate differentiation & $326(81.5)$ & $78.6 \%$ & & & \\
\hline Poorly differentiation & $37(9.25)$ & $48.8 \%$ & & & \\
\hline Undifferentiation & $4(1.00)$ & $66.7 \%$ & & & \\
\hline No. of retrieved LNs (\%) & & & 0.958 & & \\
\hline Adequate $(n \geq 12)$ & $253(63.2)$ & $74.6 \%$ & & & \\
\hline Inadequate $(\mathrm{n}<12)$ & $147(36.8)$ & $80.0 \%$ & & & \\
\hline AJCC $8^{\text {th }}$ pT stage (\%) & & & $<0.001$ & $1.52(1.12-2.06)$ & 0.008 \\
\hline pT1 & $17(4.3)$ & $100 \%$ & & & \\
\hline pT2 & $51(12.8)$ & $90.4 \%$ & & & \\
\hline pT3 & $212(53.0)$ & $78.8 \%$ & & & \\
\hline $\mathrm{pT} 4 \mathrm{a}$ & $95(23.8)$ & $71.5 \%$ & & & \\
\hline pT4b & $25(6.3)$ & $54.2 \%$ & & & \\
\hline AJCC $8^{\text {th }} \mathrm{pN}$ stage (\%) & & & $<0.001$ & $1.53(1.35-1.74)$ & $<0.001$ \\
\hline pNO & $240(60.0)$ & $90.4 \%$ & & & \\
\hline $\mathrm{pN} 1 \mathrm{a}$ & $60(15.0)$ & $77.3 \%$ & & & \\
\hline $\mathrm{pN} 1 \mathrm{~b}$ & $41(10.3)$ & $62.3 \%$ & & & \\
\hline $\mathrm{pN} 1 \mathrm{c}$ & $5(1.30)$ & $75.0 \%$ & & & \\
\hline $\mathrm{pN} 2 \mathrm{a}$ & $24(6.00)$ & $61.0 \%$ & & & \\
\hline $\mathrm{pN} 2 \mathrm{~b}$ & $30(7.50)$ & $22.6 \%$ & & & \\
\hline Vascular invasion (\%) & & & 0.832 & & \\
\hline Absence & $340(85.0)$ & $76.5 \%$ & & & \\
\hline Presence & $60(15.0)$ & $80.3 \%$ & & & \\
\hline Perineural invasion (\%) & & & 0.623 & & \\
\hline Absence & $380(95.0)$ & $78.0 \%$ & & & \\
\hline Presence & $20(5.00)$ & $77.5 \%$ & & & \\
\hline
\end{tabular}

AJCC, American Joint Committee on Cancer; Cl, confidence interval; DFS, disease-free survival; HR, hazard ratio; LNs, lymph nodes; No., number. Significant values (P < 0.05) are in bold.

neutrophil count (12), lymphocyte count (13), NLR (14), LMR (15), and PLR (16). We thus collected and analyzed information on markers previously documented to be related to the prognosis of CRC. The current findings demonstrated that neutrophil count was an independent predictor of OS. Neutrophils, as a hallmark of cancer, reflect the status of host inflammation and participate in different stages of the oncogenic processes of tumor initiation, growth, proliferation, and metastatic spread $(30,31)$. Tumor initiation can be promoted by the production of matrix metalloproteinase 9 and the release of reactive oxygen species, reactive nitrogen species, or proteases by neutrophils $(32,33)$. Neutrophils can also mediate and facilitate tumor proliferation by attenuating the immune system and via degradation of insulin receptor substrate 1 and the activation of phosphoinositide 3kinase signaling as a result of the transfer of neutrophil elastase to cancer cells $(34,35)$.

The current study indicated that neutrophils and the NLR were also independent predictors of DFS, and we hypothesized 
TABLE 4 | Univariate analysis of preoperative inflammatory and nutritional factors.

\begin{tabular}{|c|c|c|c|c|}
\hline \multirow[t]{2}{*}{ Variables } & \multicolumn{2}{|c|}{ Overall survival } & \multicolumn{2}{|c|}{ Disease-free survival } \\
\hline & HR (95\% Cl) & $P$ value & HR (95\% Cl) & $P$ value \\
\hline \multicolumn{5}{|c|}{ Inflammatory/laboratory data } \\
\hline $\operatorname{WBC}\left(\times 10^{9} / \mathrm{L}\right)$ & $1.11(1.01-1.23)$ & 0.035 & $1.11(1.01-1.21)$ & 0.025 \\
\hline Neut $\left(\times 10^{9} / \mathrm{L}\right)$ & $1.15(1.06-1.24)$ & $<0.001$ & $1.14(1.06-1.22)$ & 0.001 \\
\hline Lym (×109/L) & $0.817(0.524-1.28)$ & 0.375 & $0.723(0.471-1.11)$ & 0.139 \\
\hline Plt $\left(\times 10^{9} / \mathrm{L}\right)$ & $1.00(0.997-1.00)$ & 0.991 & $1.00(0.997-1.00)$ & 0.849 \\
\hline NWR & $1.02(1.00-1.04)$ & 0.026 & $1.02(1.00-1.04)$ & 0.024 \\
\hline NLR & $1.01(0.974-1.05)$ & 0.564 & $0.960(0.963-0.986)$ & 0.003 \\
\hline LWR & $0.961(0.933-0.989)$ & 0.007 & $1.06(1.04-1.09)$ & $<0.001$ \\
\hline PNR & $0.988(0.977-0.998)$ & 0.024 & $0.996(0.992-1.00)$ & 0.088 \\
\hline PLR & $1.00(0.998-1.00)$ & 0.995 & $1.002(1.001-1.003)$ & $<0.001$ \\
\hline \multicolumn{5}{|c|}{ Nutritional data } \\
\hline $\mathrm{Hgb}(\mathrm{g} / \mathrm{L})$ & $0.602(0.331-1.10)$ & 0.096 & $0.634(0.368-1.09)$ & 0.100 \\
\hline Alb (g/L) & $0.405(0.224-0.730)$ & 0.003 & $0.444(0.259-0.759)$ & 0.003 \\
\hline $\mathrm{BMI}\left(\mathrm{kg} / \mathrm{m}^{2}\right)$ & $0.328(0.178-0.605)$ & $<0.001$ & $0.450(0.249-0.814)$ & 0.008 \\
\hline
\end{tabular}

Alb, albumin; BMI, body mass index; Cl, confidence interval; Hgb, hemoglobin; HR, hazard ratio; LWR, lymphocyte to white blood cell ratio; Lym, lymphocyte count; Neut, neutrophil count;

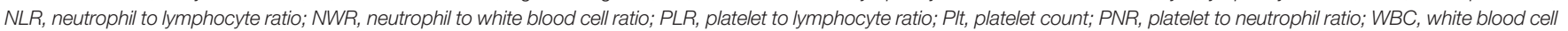
count; Hgb, $\leq 120$ vs. $>120 \mathrm{~g} / \mathrm{L} ;$ Alb, $\leq 38$ vs. $>38 \mathrm{~g} / \mathrm{L} ; \mathrm{BMl}, \leq 19.5 \mathrm{vs} .>19.5 \mathrm{~kg} / \mathrm{m}^{2}$. Significant values $(P<0.05)$ are in bold.

TABLE 5 | Prognostic performances of different models in terms of overall survival.

\begin{tabular}{|c|c|c|c|c|c|}
\hline Prognostic models & \multicolumn{2}{|c|}{ Multivariate analysis } & AUC $(95 \% \mathrm{Cl})$ & C-index & AIC \\
\hline Inflammatory/laboratory model & & & $0.796(0.720-0.872)$ & 0.794 & 522.6 \\
\hline Tumor differentiation grade & $2.56(1.58-4.15)$ & $<0.001$ & & & \\
\hline pT stage ${ }^{\#}$ & & 0.051 & & & \\
\hline WBC $\left(\times 10^{9} / \mathrm{L}\right)$ & & 0.299 & & & \\
\hline LWR & & 0.960 & & & \\
\hline PNR & & 0.178 & & & \\
\hline Nutritional model & & & $0.793(0.722-0.864)$ & 0.782 & 521.5 \\
\hline $\mathrm{pN}$ stage & $1.60(1.40-1.83)$ & $<0.001$ & & & \\
\hline Tumor differentiation grade & $2.84(1.73-4.67)$ & $<0.001$ & & & \\
\hline pT stage s $^{\#}$ & & 0.181 & & & \\
\hline pN stage & $1.60(1.40-1.83)$ & $<0.001$ & & & \\
\hline Tumor differentiation grade & $2.80(1.72-4.57)$ & $<0.001$ & & & \\
\hline pT stage ${ }^{\#}$ & & 0.161 & & & \\
\hline WBC $\left(\times 10^{9} / L\right)$ & & 0.466 & & & \\
\hline Neut $\left(\times 10^{9} / \mathrm{L}\right)$ & $1.21(1.10-1.33)$ & $<0.001$ & & & \\
\hline NWR & & 0.957 & & & \\
\hline LWR & & 0.603 & & & \\
\hline PNR & & 0.241 & & & \\
\hline $\mathrm{Hgb}(\mathrm{g} / \mathrm{L})$ & & 0.106 & & & \\
\hline Alb (g/L) & & 0.283 & & & \\
\hline $\mathrm{BMI}\left(\mathrm{kg} / \mathrm{m}^{2}\right)$ & $0.311(0.166-0.582)$ & $<0.001$ & & & \\
\hline $\begin{array}{l}\text { Control model } \\
\text { AJCC } 8^{\text {th }} \text { pTNM (pT, pN) }\end{array}$ & & & $0.789(0.716-0.862)$ & 0.776 & 535.3 \\
\hline
\end{tabular}


TABLE 6 | Prognostic performances of different models in terms of disease-free survival.

\begin{tabular}{|c|c|c|c|c|c|}
\hline \multirow[t]{2}{*}{ Prognostic models } & \multicolumn{2}{|c|}{ Multivariate analysis } & \multirow[t]{2}{*}{ AUC $(95 \% \mathrm{Cl})$} & \multirow[t]{2}{*}{ C-index } & \multirow[t]{2}{*}{ AIC } \\
\hline & HR (95\% Cl) & $P$ value & & & \\
\hline Inflammatory/laboratory model & & & $0.784(0.715-0.854)$ & 0.785 & 646.6 \\
\hline pT stage & $1.40(1.03-1.91)$ & 0.031 & & & \\
\hline pN stage & $1.55(1.37-1.76)$ & $<0.001$ & & & \\
\hline Tumor differentiation grade & $1.91(1.21-3.02)$ & 0.005 & & & \\
\hline WBC $\left(\times 10^{9} / \mathrm{L}\right)$ & & 0.545 & & & \\
\hline Neut $\left(\times 10^{9} / \mathrm{L}\right)$ & $1.11(1.01-1.22)$ & 0.032 & & & \\
\hline NWR & & 0.656 & & & \\
\hline LWR & & 0.820 & & & \\
\hline NLR & $1.04(1.01-1.08)$ & 0.014 & & & \\
\hline PNR & & 0.196 & & & \\
\hline PLR & & 0.835 & & & \\
\hline Nutritional model & & & $0.779(0.714-0.846)$ & 0.767 & 653.3 \\
\hline pT stage & & 0.059 & & & \\
\hline pN stage & $1.60(1.42-1.80)$ & $<0.001$ & & & \\
\hline Tumor differentiation grade & $2.12(1.37-3.29)$ & 0.001 & & & \\
\hline $\mathrm{Hgb}(\mathrm{g} / \mathrm{L})$ & & 0.433 & & & \\
\hline Alb (g/L) & $0.454(0.263-0.785)$ & 0.005 & & & \\
\hline BMl $\left(\mathrm{kg} / \mathrm{m}^{2}\right)$ & & 0.061 & & & \\
\hline Inflammatory and nutritional model & & & $0.803(0.738-0.869)$ & 0.777 & 648.0 \\
\hline pT stage & & 0.066 & & & \\
\hline pN stage & $1.59(1.41-1.79)$ & $<0.001$ & & & \\
\hline Tumor differentiation grade & $2.15(1.39-3.33)$ & 0.001 & & & \\
\hline WBC $\left(\times 10^{9} / \mathrm{L}\right)$ & & 0.660 & & & \\
\hline Neut $\left(\times 10^{9} / L\right)$ & $1.11(1.01-1.22)$ & 0.039 & & & \\
\hline NWR & & 0.670 & & & \\
\hline LWR & & 0.985 & & & \\
\hline NLR & $1.04(1.02-1.08)$ & 0.014 & & & \\
\hline PNR & & 0.232 & & & \\
\hline PLR & & 0.745 & & & \\
\hline $\mathrm{Hgb}(\mathrm{g} / \mathrm{L})$ & & 0.579 & & & \\
\hline Alb (g/L) & $0.522(0.293-0.926)$ & 0.027 & & & \\
\hline BMl $\left(\mathrm{kg} / \mathrm{m}^{2}\right)$ & & 0.055 & & & \\
\hline Control model & & & $0.784(0.718-0.850)$ & 0.764 & 668.4 \\
\hline AJCC $8^{\text {th }}$ pTNM (pT, pN) & & & & & \\
\hline
\end{tabular}

AJCC, American Joint Committee on Cancer; AIC, Akaike's Information Criterion; Alb, albumin; BMI, body mass index;

$\mathrm{Cl}$, confidence interval; C-index, concordance index; Hgb, hemoglobin; HR, hazard ratio; LWR, lymphocyte to white blood cell ratio;

$N L R$, neutrophil to lymphocyte ratio; NWR, neutrophil to white blood cell ratio; PLR, platelet to lymphocyte ratio;

$P N R$, platelet to neutrophil ratio; WBC, white blood cell count;

$\mathrm{Hgb}, \leq 120$ vs. $>120 \mathrm{~g} / \mathrm{L} ;$ Alb, $\leq 38 \mathrm{vs.}>38 \mathrm{~g} / \mathrm{L} ; \mathrm{BMI}, \leq 19.5 \mathrm{vs} .>19.5 \mathrm{~kg} / \mathrm{m}^{2}$. Significant values $(P<0.05)$ are in bold.

that they might be associated with tumor recurrence in patients with CRC. Neutrophils can facilitate metastatic spread by inhibiting natural killer function and promoting the extravasation of tumor cells (36). An elevated NLR indicates a systemic inflammatory status, which, in turn, suggests neutrophilia, lymphocytopenia, or a combination of both. The tumor phenotype can also promote the influx of inflammatory lymphocytes around the tumor leading to cell destruction within the surrounding tissues, thereby generating a broader nonspecific inflammatory response (37). In addition, lymphocytopenia may lead to a poorer lymphocyte-mediated immune response to malignancy, and a subsequently increased potential for tumor recurrence (38).

Previous studies showed an association between preoperative nutritional status and mortality in patients with gastrointestinal cancers $(17,18)$. Here, we attempted to apply objective and easily measurable markers of nutritional status, and found that underweight patients had significantly poorer OS and BMI was identified as an independent predictive factor of OS. These findings were consistent with previous studies $(39,40)$, which showed that BMI was associated with prognosis (40), and a high metabolic rate and anorexia induced by cancer may lead to patients being underweight (39). Furthermore, cytokine responses and subsequent immune system activation were impaired in patients with chronic malnutrition and micronutrient deficiency, potentially influencing interactions between the tumor and immune system (41). Tumor cells have been shown to interact with circulating immune cells via various molecular signals, from initial carcinogenesis to metastasis (42).

Serum albumin was identified as an independent predictor of DFS in patients with CRC in the current study. Although serum albumin cannot comprehensively reflect the patient's nutritional status, it has nevertheless been used extensively and is accepted as a good indicator of nutritional status (43). Serum albumin was also reported to play a potentially important protective role in promoting the removal of reactive oxygen species, which is a process related to the pathogenesis of many diseases, including cancers (44). Cancer-related systemic inflammation, 
A

C ROC for OS

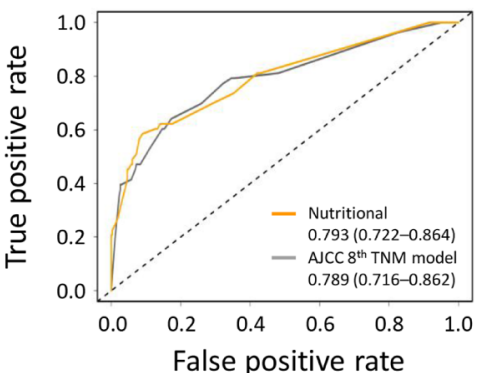

E ROC for OS

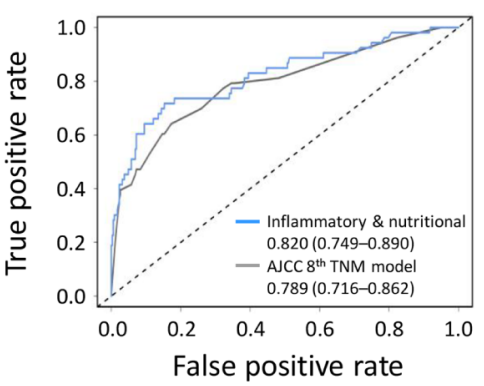

B ROC for DFS

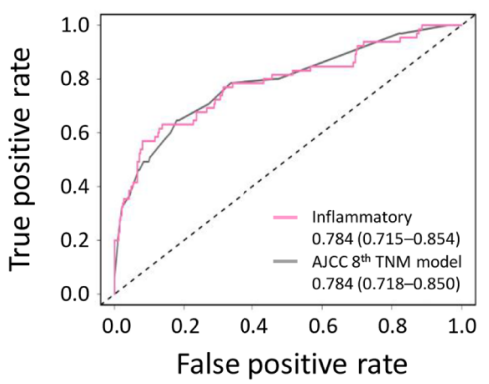

D ROC for DFS

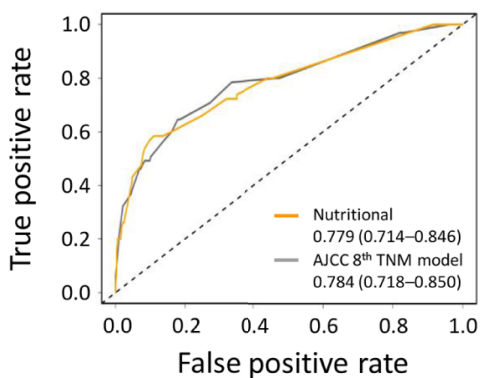

F ROC for DFS

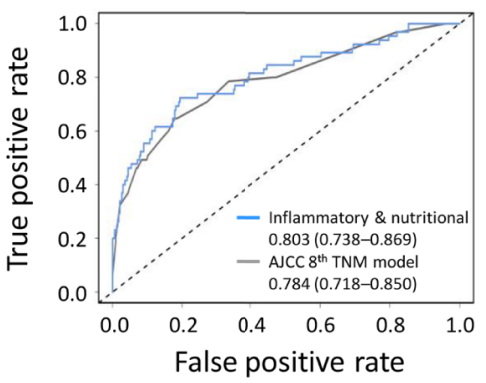

FIGURE 1 | Receiver operating characteristic curves $(\mathrm{ROC})$ comparing discriminative abilities of novel prognostic models with the American Joint Committee on Cancer (AJCC) 8th TNM classification. (A) Inflammatory model versus AJCC 8th TNM classification for overall survival (OS); (B) inflammatory model versus AJCC 8th TNM classification for disease-free survival (DFS); (C) nutritional model versus AJCC 8th TNM classification for OS; (D) nutritional model versus AJCC 8th TNM classification for DFS; (E) combined inflammatory and nutritional model versus AJCC 8th TNM classification for OS; (F) combined inflammatory and nutritional model versus AJCC 8th TNM classification for DFS.

malnutrition, and an increased turnover of albumin by tumors can result in the inhibition of albumin synthesis and a reduction in serum albumin (45). The relationship between serum albumin and survival might also be influenced by an elevation in cytokines (46). C-reactive protein, tumor necrosis factor, and interleukin-1 are involved in the pathogenesis of cancer, and can decrease serum albumin concentrations $(46,47)$. Alternatively, tumor necrosis factor may increase the permeability of the microvasculature, thus allowing the increased transcapillary passage of albumin and leading to low levels of serum albumin. Serum interleukin-6 is also increased in acute and chronic inflammatory situations and has been shown to be associated with hypoalbuminemia (48).

Nutritional status can also be a marker of systemic inflammatory responses, and some studies have demonstrated a relationship between acute-phase reactants and survival in patients with cancer. They indicated that hypoalbuminemia was a marker of malnutrition or systemic inflammation, because some proinflammatory substances, such as cytokines, reduced the concentration of albumin $(49,50)$. Furthermore, significant inflammation or injury can influence appetite, gastrointestinal motility, and hemodynamic stability, which may, in turn, affect the nutritional status of patients (51).

Many prognostic models incorporating different factors have been developed to predict the prognosis or risk of death in patients with CRC. Bibault et al. developed and validated a model incorporating tumor features and patient medical and demographic information to predict survival in CRC, with a high predictive performance (AUC, 0.84; accuracy, 0.83) at the individual scale (52). Dienstmann et al. confirmed the 
A

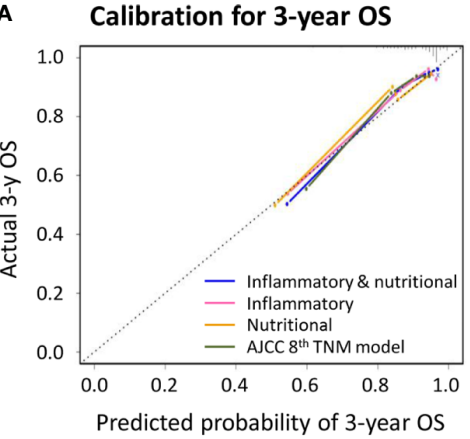

C

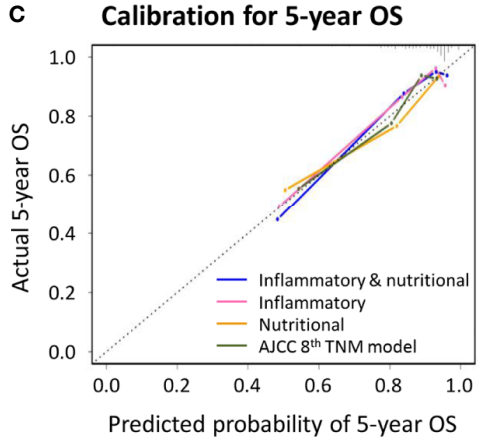

B Calibration for 3-year DFS

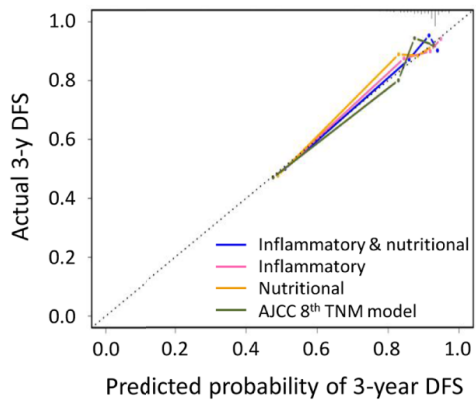

D Calibration for 5-year DFS

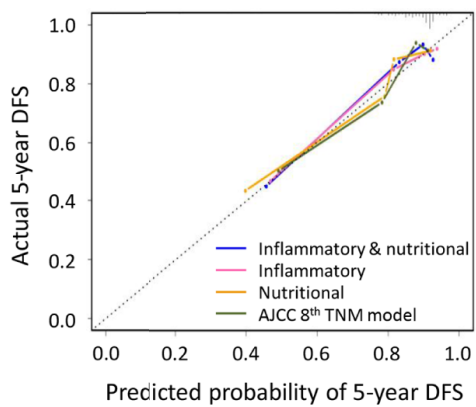

FIGURE 2 | Calibration curves for novel prognostic models and the American Joint Committee on Cancer (AJCC) 8th TNM classification for predicting (A) 3-year overall survival (OS); (B) 3-year disease-free survival (DFS); (C) 5-year OS; and (D) 5-year DFS.
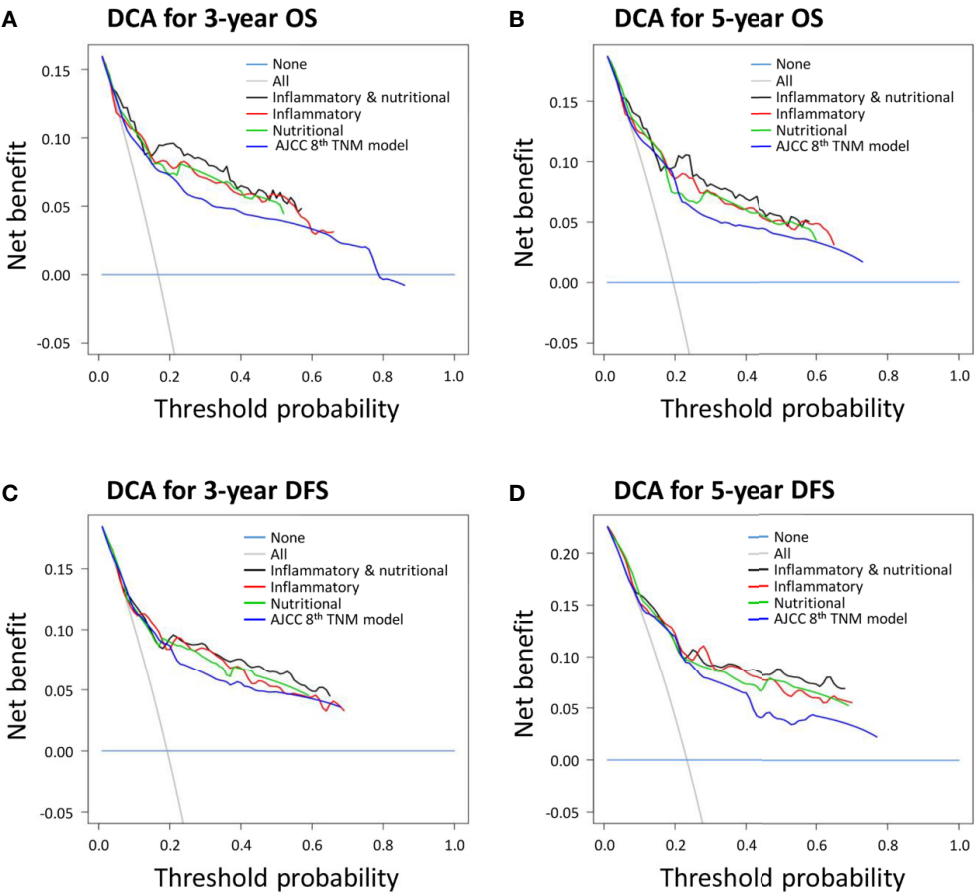

D DCA for 5-year DFS

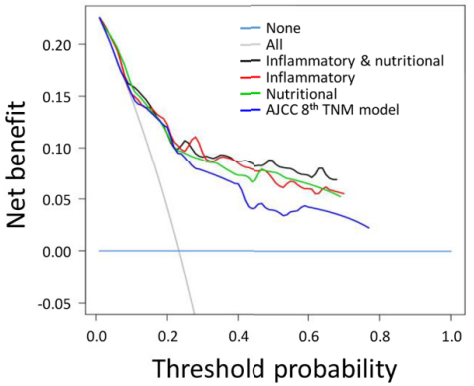

FIGURE 3 | Decision curve analyses (DCAs) of novel prognostic models and the American Joint Committee on Cancer (AJCC) 8th TNM classification for (A) 3-year overall survival (OS); (B) 3-year disease-free survival (DFS); (C) 5-year OS; and (D) 5-year DFS. 


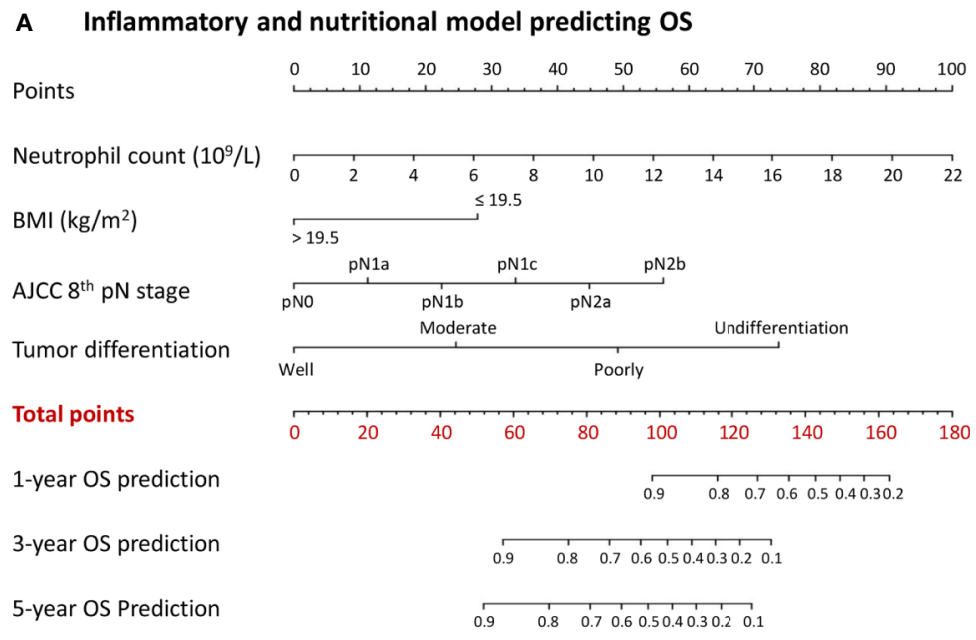

B Inflammatory and nutritional model predicting DFS

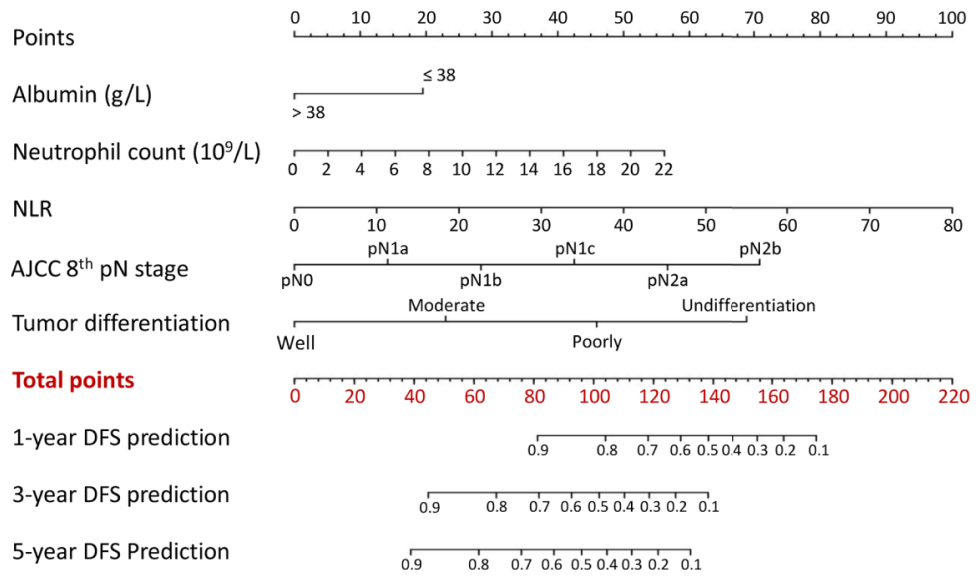

FIGURE 4 | Nomograms conveying the results of the novel prognostic models incorporating clinicopathological characteristics and preoperative inflammatory and nutritional factors for predicting (A) overall survival (OS) and (B) disease-free survival (DFS) in patients with colorectal cancer. BMI, body mass index; NLR, neutrophillymphocyte ratio.

importance of genomic markers, transcriptomic subtyping, and microenvironmental features for survival prediction in patients with stage II/III CRC $(53,54)$. Importantly, establishment of the nomogram improved the individual prediction of survival outcomes for patients with CRC $(55,56)$.

We developed several novel prognostic models incorporating the nutritional and inflammatory prognostic factors identified in this study. The combined inflammatory and nutritional prognostic model showed superior model discriminative ability, better modelfitting performance, and higher net benefits compared with either the inflammatory or nutritional model alone. The AJCC 8th TNM classification is considered to be the gold standard for prognostic prediction in patients with CRC. However, the present study demonstrated that the novel combined inflammatory and nutritional prognostic model also had superior predictive performances for OS and DFS compared with the AJCC 8th TNM classification. A nomogram is a visible tool for individually predicting survival outcomes, with high predictive accuracy and comprehensive outcomes in cancer (57). Nomograms may help clinicians to predict individual survival outcomes for patients with CRC after curative surgery, and may provide useful information in terms of recommending postoperative adjuvant therapy or intensive follow-up. Importantly, the markers required for this novel prognostic nomogram model could be easily obtained, making it a convenient tool for optimally predicting survival outcomes and facilitating decision making.

The results of studies concerning the ability of preoperative or perioperative nutritional supplementation to improve postoperative outcomes are controversial. European guidelines for surgical patients support the use of preoperative nutritional supplementation in severely malnourished patients, even if it delays surgery (58). Moreover, a previous study demonstrated that preoperative oral arginine and n-3 fatty acid supplementation improved the immunometabolic host response and outcomes after surgery for 


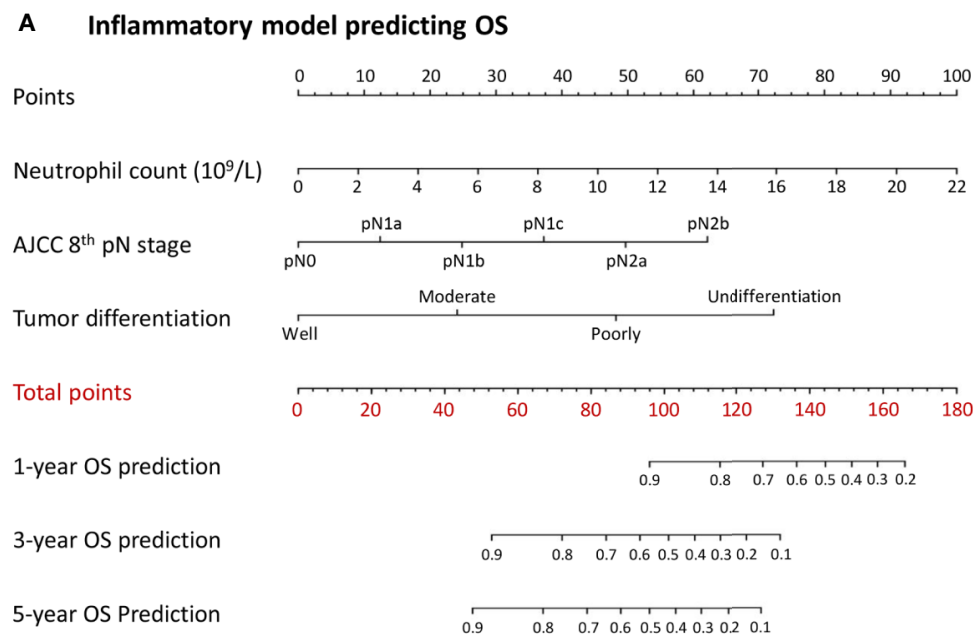

\section{B Inflammatory model predicting DFS}

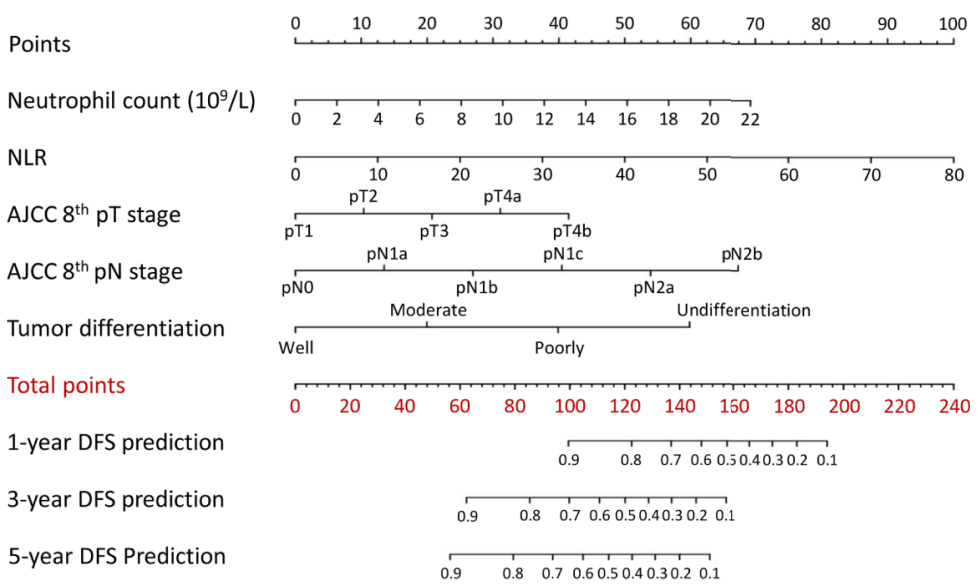

FIGURE 5 | Nomograms conveying the results of the novel prognostic models incorporating clinicopathological characteristics and preoperative inflammatory factors for predicting (A) overall survival (OS) and (B) disease-free survival (DFS) in patients with colorectal cancer. NLR, neutrophil-lymphocyte ratio.

CRC (59), while a more recent study proved that protein intake after colorectal surgery was associated with reduced length of hospital stay (60). However, some randomized control trials failed to demonstrate any postoperative benefits from perioperative nutrient therapy, including oral omega-3 fatty acids, eicosapentaenoic acid, or docosahexaenoic acid intake, in patients with CRC surgery $(61,62)$. Whether or not perioperative nutritional intervention can improve a patient's long-term outcome thus remains unclear, and further studies are still required to clarify this issue.

The strength of this study was identifying the prognostic role of inflammatory and nutritional status in predicting survival based on standard laboratory factors that are routinely applied in clinical practice. However, the study had some limitations. First, selection bias may have occurred because of the retrospective, single-center nature of the study, and the main findings therefore need external validation in prospective multicenter studies with larger populations. Second, we were not able to include some important confounding factors, including KRAS, BRAF, or microsatellite instability. Third, the examined lymph nodes in some patients in this study did not meet the minimum 12 lymph nodes requirements of the guidelines, which may have affected the TNM staging. Further verification is therefore required. Finally, although the difference between the novel prediction model and the AJCC 8th TNM classification of CRC was statistically significant, the absolute AUC values were still not qualified for clinical recommendations. More studies are therefore required to validate the main findings of the current study.

\section{CONCLUSION}

In summary, preoperative nutritional and inflammatory factors have significant prognostic values in patients with CRC. A novel prognostic model incorporating both preoperative inflammatory 


\section{A Nutritional model predicting OS}

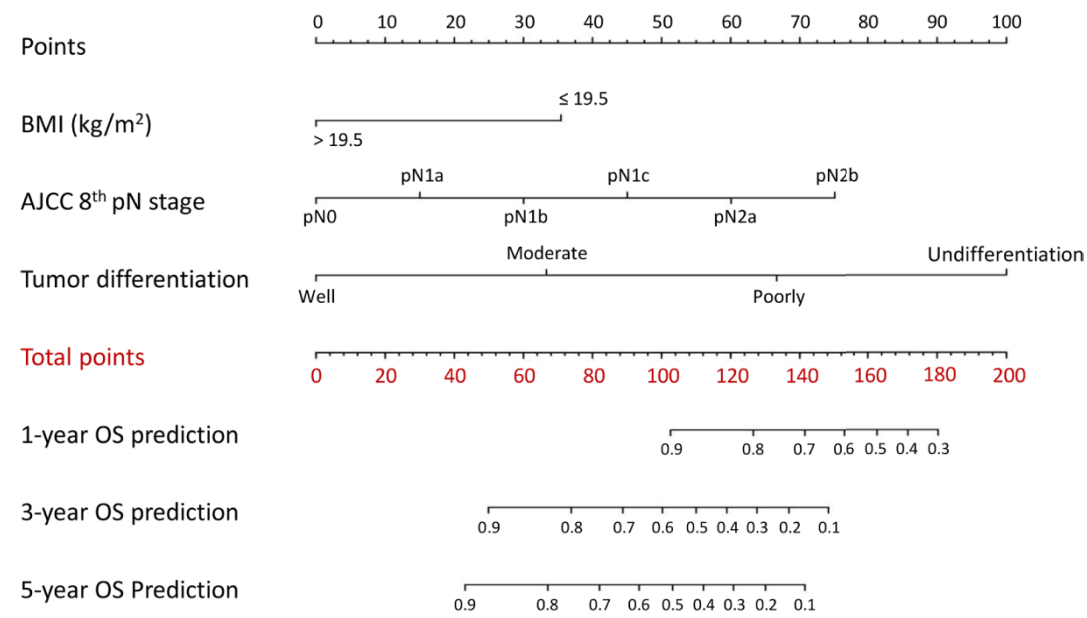

B Nutritional model predicting DFS

Points

$\begin{array}{lllllllllll}0 & 10 & 20 & 30 & 40 & 50 & 60 & 70 & 80 & 90 & 100\end{array}$

Albumin (g/L)

AJCC $8^{\text {th }} \mathrm{pN}$ stage

Tumor differentiation

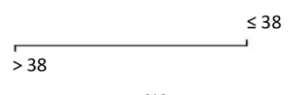

38

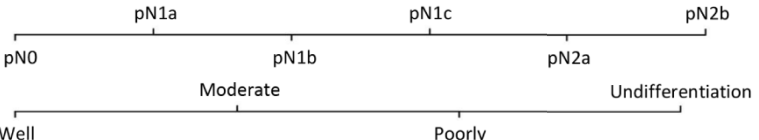

Total points

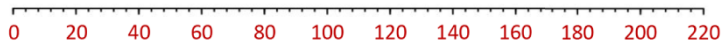

1-year DFS prediction

0.9

3-year DFS prediction

\begin{tabular}{llllllllll}
\hline 0.9 & 0.8 & 0.7 & 0.6 & 0.5 & 0.4 & 0.3 & 0.2 & 0.1
\end{tabular}

5-year DFS Prediction

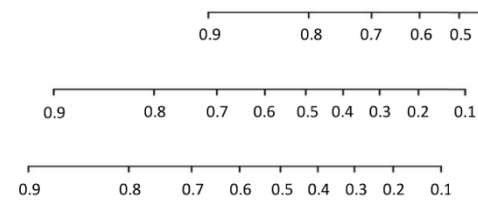

FIGURE 6 | Nomograms conveying the results of the novel prognostic models incorporating clinicopathological characteristics and preoperative nutritional factors for predicting (A) overall survival (OS) and (B) disease-free survival (DFS) in patients with colorectal cancer. BMl, body mass index; NLR, neutrophil-lymphocyte ratio.

and nutritional markers provided better prognostic performance than the AJCC 8th TNM classification. The novel developed nomogram incorporating preoperative inflammatory and nutritional markers could individually predict OS and DFS in patients with CRC. These results suggested the need to raise awareness of the importance of preoperative inflammatory and nutritional statuses in patients with CRC. However, the main findings of current study need to be interpreted with caution and require external validation in future studies.

\section{DATA AVAILABILITY STATEMENT}

The raw data supporting the conclusions of this article will be made available by the authors, without undue reservation.

\section{ETHICS STATEMENT}

The studies involving human participants were reviewed and approved by Ethics Committee of the Cancer Hospital of China Medical University, Liaoning Cancer Hospital and Institute. The patients/participants provided their written informed consent to participate in this study.

\section{AUTHOR CONTRIBUTIONS}

Study concept and design: NZ, FN, RG, and CZ. Acquisition of data: NZ, FN, RG, RZ, and CZ. Analysis and interpretation of data: NZ, FN, RG, RZ, and CZ. Drafting of the manuscript: NZ, 
FN, RG, JP, YQ, JF, BJ, YL, ZC, ZM, MA, JZ, RZ, and CZ. Critical revision of the manuscript for important intellectual content: NZ, JZ, RZ, and CZ. Obtained funding: CZ and ZM. Lead corresponding author: CZ. All authors contributed to the article and approved the submitted version.

\section{REFERENCES}

1. Siegel RL, Miller KD, Jemal A. Cancer statistics, 2019. CA Cancer J Clin (2019) 69(1):7-34. doi: 10.3322/caac.21551

2. Bray F, Ferlay J, Soerjomataram I, Siegel RL, Torre LA, Jemal A. Global cancer statistics 2018: GLOBOCAN estimates of incidence and mortality worldwide for 36 cancers in 185 countries. CA Cancer J Clin (2018) 68(6):394-424. doi: $10.3322 /$ caac. 21492

3. Amin MB, Edge SB. AJCC cancer staging manual. 8th ed. springer (2017). Available at: https://www.springer.com/us/book/9783319406176\#aboutBook.

4. Sasaki K, Morioka D, Conci S, Margonis GA, Sawada Y, Ruzzenente A, et al. The tumor burden score: a new "Metro-ticket" prognostic tool for colorectal liver metastases based on tumor size and number of tumors. Ann Surg (2018) 267(1):132-41. doi: 10.1097/SLA.0000000000002064

5. Wang L, Lo CH, He X, Hang D, Wang M, Wu K, et al. Risk factor profiles differ for cancers of different regions of the colorectum. Gastroenterology (2020) S0016-5085(20):30408-X. doi: 10.1053/j.gastro.2020.03.054

6. Nagtegaal ID, Knijn N, Hugen N, Marshall HC, Sugihara K, Tot T, et al. Tumor deposits in colorectal cancer: improving the value of modern staging-a systematic review and meta-analysis. J Clin Oncol (2017) 35(10):1119-27. doi: 10.1200/JCO.2016.68.9091

7. Rosenberg R, Engel J, Bruns C, Heitland W, Hermes N, Jauch KW, et al. The prognostic value of lymph node ratio in a population-based collective of colorectal cancer patients. Ann Surg (2010) 251(6):1070-8. doi: 10.1097/ SLA.0b013e3181d7789d

8. Rönnow CF, Arthursson V, Toth E, Krarup PM, Syk I, Thorlacius H. Lymphovascular infiltration, not depth of invasion, is the critical risk factor of metastases in early colorectal cancer: retrospective population-based cohort study on prospectively collected data, including validation. Ann Surg (2020). doi: 10.1097/SLA.0000000000003854. [Epub ahead of print].

9. Holch JW, Ricard I, Stintzing S, Fischer von Weikersthal L, Decker T, Kiani A, et al. Relevance of baseline carcinoembryonic antigen for first-line treatment against metastatic colorectal cancer with FOLFIRI plus cetuximab or bevacizumab (FIRE-3 trial). Eur J Cancer (2019) 106:115-25. doi: 10.1016/ j.ejca.2018.10.001

10. Suzuki Y, Okabayashi K, Hasegawa H, Tsuruta M, Shigeta K, Kondo T, et al. Comparison of Preoperative Inflammation-based Prognostic Scores in Patients With Colorectal Cancer. Ann Surg (2018) 267(3):527-31. doi: 10.1097/SLA.0000000000002115

11. Malietzis G, Giacometti M, Askari A, Nachiappan S, Kennedy RH, Faiz OD, et al. A preoperative neutrophil to lymphocyte ratio of 3 predicts disease-free survival after curative elective colorectal cancer surgery. Ann Surg (2014) 260 (2):287-92. doi: 10.1097/SLA.0000000000000216

12. Diefenhardt M, Hofheinz RD, Martin D, Beißbarth T, Arnold D, Hartmann A, et al. Leukocytosis and neutrophilia as independent prognostic immunological biomarkers for clinical outcome in the CAO/ARO/AIO-04 randomized phase 3 rectal cancer trial. Int J Cancer (2019) 145(8):2282-91. doi: 10.1002/ ijc. 32274

13. Iseki Y, Shibutani M, Maeda K, Nagahara H, Tamura T, Ohira G, et al. The impact of the preoperative peripheral lymphocyte count and lymphocyte percentage in patients with colorectal cancer. Surg Today (2017) 47(6):743-54. doi: 10.1007/s00595-016-1433-2

14. Wang SC, Chou JF, Strong VE, Brennan MF, Capanu M, Coit DG. Pretreatment neutrophil to lymphocyte ratio independently predicts disease-specific survival in resectable gastroesophageal junction and gastric adenocarcinoma. Ann Surg (2016) 263(2):292-7. doi: 10.1097/SLA.0000000000001189

15. Chan JC, Chan DL, Diakos CI, Engel A, Pavlakis N, Gill A, et al. The lymphocyte-to-monocyte ratio is a superior predictor of overall survival in comparison to established biomarkers of resectable colorectal cancer. Ann Surg (2017) 265(3):539-46. doi: 10.1097/SLA.0000000000001743

\section{FUNDING}

This work was supported, in part, by the China Scholarship Council (201908050148) and National Natural Science Foundation of China (81774112).

16. Russo A, Russano M, Franchina T, Migliorino MR, Aprile G, Mansueto G, et al. Prognostic significance of neutrophil-to-lymphocyte ratio (NLR) and platelet-to-lymphocyte ratio (PLR) in non-small cell lung cancer (NSCLC) treated with immune checkpoint inhibitors. Adv Ther (2020) 37(3):1145-55. doi: 10.1007/s12325-020-01229-w

17. Schwegler I, von Holzen A, Gutzwiller JP, Schlumpf R, Mühlebach S, Stanga Z. Nutritional risk is a clinical predictor of postoperative mortality and morbidity in surgery for colorectal cancer. Br J Surg (2010) 97(1):92-7. doi: $10.1002 /$ bjs.6805

18. Reisinger KW, van Vugt JL, Tegels JJ, Snijders C, Hulsewé KW, Hoofwijk AG, et al. Functional compromise reflected by sarcopenia, frailty, and nutritional depletion predicts adverse postoperative outcome after colorectal cancer surgery. Ann Surg (2015) 261(2):345-52. doi: 10.1097/SLA.0000000000000628

19. Van Blarigan EL, Fuchs CS, Niedzwiecki D, Zhang S, Saltz LB, Mayer RJ, et al. Association of survival with adherence to the American cancer society nutrition and physical activity guidelines for cancer survivors after colon cancer diagnosis: The CALGB 89803/Alliance Trial. JAMA Oncol (2018) 4 (6):783-90. doi: 10.1001/jamaoncol.2018.0126

20. Hennessey DB, Burke JP, Ni-Dhonochu T, Shields C, Winter DC, Mealy K. Preoperative hypoalbuminemia is an independent risk factor for the development of surgical site infection following gastrointestinal surgery: a multi-institutional study. Ann Surg (2010) 252(2):325-9. doi: 10.1097/ SLA.0b013e3181e9819a

21. Okuno M, Goumard C, Kopetz S, Vega EA, Joechle K, Mizuno T, et al. Loss of muscle mass during preoperative chemotherapy as a prognosticator for poor survival in patients with colorectal liver metastases. Surgery (2019) 165 (2):329-36. doi: 10.1016/j.surg.2018.07.031

22. Luvián-Morales J, González-Trejo S, Carrillo JF, Herrera-Goepfert R, AielloCrocifoglio V, Gallardo-Rincón D, et al. Association of the prognostic nutritional index and overall survival in patients with colorectal cancer: A STROBE compliant retrospective cohort study. Cancer Med (2019) 8(7):337988. doi: $10.1002 /$ cam 4.2212

23. de Gramont A, Hubbard J, Shi Q, O’Connell MJ, Buyse M, Benedetti J, et al. Association between disease-free survival and overall survival when survival is prolonged after recurrence in patients receiving cytotoxic adjuvant therapy for colon cancer: simulations based on the 20,800 patient ACCENT data set. J Clin Oncol (2010) 28(3):460-5. doi: 10.1200/JCO.2009.23.1407

24. Soumerai JD, Ni A, Darif M, Londhe A, Xing G, Mun Y, et al. Prognostic risk score for patients with relapsed or refractory chronic lymphocytic leukaemia treated with targeted therapies or chemoimmunotherapy: a retrospective, pooled cohort study with external validations. Lancet Haematol (2019) 6(7): e366-74. doi: 10.1016/S2352-3026(19)30085-7

25. Wang J, Song Y, Liu X, Jin J, Wang W, Yu Z, et al. Comparison of outcome and toxicity of postoperative intensity-modulated radiation therapy with two-dimensional radiotherapy in patients with soft tissue sarcoma of extremities and trunk. Cancer Med (2019) 8(3):902-9. doi: 10.1002/ cam4.1919

26. Alba AC, Agoritsas T, Walsh M, Hanna S, Iorio A, Devereaux PJ, et al. Discrimination and calibration of clinical prediction models: users' guides to the medical literature. JAMA (2017) 318(14):1377-84. doi: 10.1001/ jama.2017.12126

27. Fitzgerald M, Saville BR, Lewis RJ. Decision curve analysis. JAMA (2015) 313 (4):409-10. doi: 10.1001/jama.2015.37

28. Mantovani A, Allavena P, Sica A, Balkwill F. Cancer-related inflammation. Nature (2008) 454(7203):436-44. doi: 10.1038/nature07205

29. Diakos CI, Charles KA, McMillan DC, Clarke SJ. Cancer-related inflammation and treatment effectiveness. Lancet Oncol (2014) 15(11): e493-503. doi: 10.1016/S1470-2045(14)70263-3

30. Hanahan D, Weinberg RA. Hallmarks of cancer: the next generation. Cell (2011) 144(5):646-74. doi: 10.1016/j.cell.2011.02.013 
31. Coffelt SB, Wellenstein MD, de Visser KE. Neutrophils in cancer: neutral no more. Nat Rev Cancer (2016) 16(7):431-46. doi: 10.1038/nrc.2016.52

32. Antonio N, Bønnelykke-Behrndtz ML, Ward LC, Collin J, Christensen IJ, Steiniche $\mathrm{T}$, et al. The wound inflammatory response exacerbates growth of pre-neoplastic cells and progression to cancer. EMBO J (2015) 34(17):221936. doi: $10.15252 / \mathrm{embj} .201490147$

33. Shojaei F, Wu X, Zhong C, Yu L, Liang XH, Yao J, et al. Bv8 regulates myeloidcell-dependent tumour angiogenesis. Nature (2007) 450(7171):825-31. doi: 10.1038/nature06348

34. Bodogai M, Moritoh K, Lee-Chang C, Hollander CM, Sherman-Baust CA, Wersto RP, et al. Immunosuppressive and prometastatic functions of myeloid-derived suppressive cells rely upon education from tumorassociated B cells. Cancer Res (2015) 75(17):3456-65. doi: 10.1158/00085472.CAN-14-3077

35. Houghton AM, Rzymkiewicz DM, Ji H, Gregory AD, Egea EE, Metz HE, et al. Neutrophil elastase-mediated degradation of IRS-1 accelerates lung tumor growth. Nat Med (2010) 16(2):219-23. doi: 10.1038/nm.2084

36. Spiegel A, Brooks MW, Houshyar S, Reinhardt F, Ardolino M, Fessler E, et al. Neutrophils suppress intraluminal NK cell-mediated tumor cell clearance and enhance extravasation of disseminated carcinoma cells. Cancer Discovery (2016) 6(6):630-49. doi: 10.1158/2159-8290.CD-15-1157

37. Coussens LM, Werb Z. Inflammation and cancer. Nature (2002) 420 (6917):860-7. doi: 10.1038/nature01322

38. Halazun KJ, Aldoori A, Malik HZ, Al-Mukhtar A, Prasad KR, Toogood GJ, et al. Elevated preoperative neutrophil to lymphocyte ratio predicts survival following hepatic resection for colorectal liver metastases. Eur J Surg Oncol (2008) 34(1):55-60. doi: 10.1016/j.ejso.2007.02.014

39. Sinicrope FA, Foster NR, Yothers G, Benson A, Seitz JF, Labianca R, et al. Body mass index at diagnosis and survival among colon cancer patients enrolled in clinical trials of adjuvant chemotherapy. Cancer (2013) 119 (8):1528-36. doi: $10.1002 / \mathrm{cncr} .27938$

40. Moon HG, Han W, Noh DY. Underweight and breast cancer recurrence and death: a report from the Korean Breast Cancer Society. J Clin Oncol (2009) 27 (35):5899-905. doi: 10.1200/JCO.2009.22.4436

41. Cunningham-Rundles S, McNeeley DF, Moon A. Mechanisms of nutrient modulation of the immune response. J Allergy Clin Immunol (2005) 115 (6):1119-28. doi: 10.1016/j.jaci.2005.04.036

42. de Visser KE, Eichten A, Coussens LM. Paradoxical roles of the immune system during cancer development. Nat Rev Cancer (2006) 6(1):24-37. doi: $10.1038 / \mathrm{nrc1} 182$

43. Franch-Arcas G. The meaning of hypoalbuminaemia in clinical practice. Clin Nutr (2001) 20(3):265-9. doi: 10.1054/clnu.2001.0438

44. Ko WF, Helzlsouer KJ, Comstock GW. Serum albumin, bilirubin, and uric acid and the anatomic site-specific incidence of colon cancer. J Natl Cancer Inst (1994) 86(24):1874-5. doi: 10.1093/jnci/86.24.1874

45. Gupta D, Lis CG. Pretreatment serum albumin as a predictor of cancer survival: a systematic review of the epidemiological literature. Nutr J (2010) 9:69. doi: 10.1186/1475-2891-9-69

46. Oñate-Ocaña LF, Aiello-Crocifoglio V, Gallardo-Rincón D, Herrera-Goepfert R, Brom-Valladares R, Carrillo JF, et al. Serum albumin as a significant prognostic factor for patients with gastric carcinoma. Ann Surg Oncol (2007) 14(2):381-9. doi: 10.1245/s10434-006-9093-x

47. Chojkier M. Inhibition of albumin synthesis in chronic diseases: molecular mechanisms. J Clin Gastroenterol (2005) 39(4 Suppl 2):S143-6. doi: 10.1097/ 01.mcg.0000155514.17715.39

48. Ashizawa T, Okada R, Suzuki Y, Takagi M, Yamazaki T, Sumi T, et al. Clinical significance of interleukin-6 (IL-6) in the spread of gastric cancer: role of IL-6 as a prognostic factor. Gastric Cancer (2005) 8(2):124-31. doi: 10.1007/ s10120-005-0315-x

49. Chang Y, An H, Xu L, Zhu Y, Yang Y, Lin Z, et al. Systemic inflammation score predicts postoperative prognosis of patients with clear-cell renal cell carcinoma. Br J Cancer (2015) 113(4):626-33. doi: 10.1038/bjc.2015.241

50. Suzuki S, Akiyoshi T, Oba K, Otsuka F, Tominaga T, Nagasaki T, et al. Comprehensive comparative analysis of prognostic value of systemic inflammatory biomarkers for patients with stage II/III colon cancer. Ann Surg Oncol (2020) 27(3):844-52. doi: 10.1245/s10434-019-07904-9
51. McSorley ST, Watt DG, Horgan PG, McMillan DC. Postoperative systemic inflammatory response, complication severity, and survival following surgery for colorectal cancer. Ann Surg Oncol (2016) 23(9):2832-40. doi: 10.1245/ s10434-016-5204-5

52. Bibault JE, Chang DT, Xing L. Development and validation of a model to predict survival in colorectal cancer using a gradient-boosted machine. Gut (2020). doi: 10.1136/gutjnl-2020-321799. gutjnl-2020-321799.

53. Dienstmann R, Mason MJ, Sinicrope FA, Phipps AI, Tejpar S, Nesbakken A, et al. Prediction of overall survival in stage II and III colon cancer beyond TNM system: a retrospective, pooled biomarker study. Ann Oncol (2017) 28 (5):1023-31. doi: 10.1093/annonc/mdx052

54. Dienstmann R, Villacampa G, Sveen A, Mason MJ, Niedzwiecki D, Nesbakken A, et al. Relative contribution of clinicopathological variables, genomic markers, transcriptomic subtyping and microenvironment features for outcome prediction in stage II/III colorectal cancer. Ann Oncol (2019) 30 (10):1622-9. doi: 10.1093/annonc/mdz287

55. Fernández Montes A, López López C, Argilés Martínez G, Páez López D, López Muñoz AM, García Paredes B, et al. Prognostic Nomogram and Patterns of Use of FOLFIRI-Aflibercept in Advanced Colorectal Cancer: A Real-World Data Analysis. Oncologist (2019) 24(8):e687-95. doi: 10.1634/ theoncologist.2018-0824

56. Sjoquist KM, Renfro LA, Simes RJ, Tebbutt NC, Clarke S, Seymour MT, et al. Personalizing Survival Predictions in Advanced Colorectal Cancer: The ARCAD Nomogram Project. J Natl Cancer Inst (2018) 110(6):638-48. doi: $10.1093 /$ jnci/djx253

57. Wei JH, Feng ZH, Cao Y, Zhao HW, Chen ZH, Liao B, et al. Predictive value of single-nucleotide polymorphism signature for recurrence in localised renal cell carcinoma: a retrospective analysis and multicentre validation study. Lancet Oncol (2019) 20(4):591-600. doi: 10.1016/S1470-2045(18)30932-X

58. Weimann A, Braga M, Harsanyi L, Laviano A, Ljungqvist O, Soeters $P$, et al. ESPEN Guidelines on Enteral Nutrition: Surgery including organ transplantation. Clin Nutr (2006) 25(2):224-44. doi: 10.1016/j.clnu.2006. 01.015

59. Braga M, Gianotti L, Vignali A, Carlo VD. Preoperative oral arginine and n-3 fatty acid supplementation improves the immunometabolic host response and outcome after colorectal resection for cancer. Surgery (2002) 132(5):805-14. doi: $10.1067 / \mathrm{msy} .2002 .128350$

60. Yeung SE, Hilkewich L, Gillis C, Heine JA, Fenton TR. Protein intakes are associated with reduced length of stay: a comparison between Enhanced Recovery After Surgery (ERAS) and conventional care after elective colorectal surgery. Am J Clin Nutr (2017) 106(1):44-51. doi: 10.3945/ajcn.116.148619

61. Sorensen LS, Thorlacius-Ussing O, Rasmussen HH, Lundbye-Christensen S, Calder PC, Lindorff-Larsen K, et al. Effects of perioperative supplementation with omega- 3 fatty acids on leukotriene $\mathrm{B}_{4}$; and leukotriene $\mathrm{B}_{5}$; production by stimulated neutrophils in patients with colorectal cancer: a randomized, placebo-controlled intervention trial. Nutrients (2014) 6(10):4043-57. doi: 10.3390/nu6104043

62. Sorensen LS, Thorlacius-Ussing O, Schmidt EB, Rasmussen HH, LundbyeChristensen S, Calder PC, et al. Randomized clinical trial of perioperative omega-3 fatty acid supplements in elective colorectal cancer surgery. Br J Surg (2014) 101(2):33-42. doi: 10.1002/bjs.9361

Conflict of Interest: The authors declare that the research was conducted in the absence of any commercial or financial relationships that could be construed as a potential conflict of interest.

The reviewer NP declared a shared affiliation with several of the authors JF, JZ, to the handling editor at the time of review.

Copyright (c) 2020 Zhang, Ning, Guo, Pei, Qiao, Fan, Jiang, Liu, Chi, Mei, Abe, Zhu, Zhang and Zhang. This is an open-access article distributed under the terms of the Creative Commons Attribution License (CC BY). The use, distribution or reproduction in other forums is permitted, provided the original author(s) and the copyright owner(s) are credited and that the original publication in this journal is cited, in accordance with accepted academic practice. No use, distribution or reproduction is permitted which does not comply with these terms. 